\title{
The relationship of body composition to daily physical activity in free-living Japanese adult men
}

\author{
Jonghoon Park $^{1,2}$, Kazuko Ishikawa-Takata ${ }^{2 *}$, Shigeho Tanaka ${ }^{3}$, Yuki Hikihara ${ }^{4}$, Kazunori Ohkawara ${ }^{5}$, \\ Shaw Watanabe ${ }^{6}$, Motohiko Miyachi $^{7}$, Akemi Morita ${ }^{8}$, Naomi Aiba ${ }^{9}$ and Izumi Tabata ${ }^{10}$ \\ ${ }^{1}$ Department of Physical Education, Konkuk University, Seoul, Korea \\ ${ }^{2}$ Department of Nutritional Education, National Institute of Health and Nutrition, 1-23-1 Toyama, \\ Shinjuku-ku, Tokyo 162-8636, Japan \\ ${ }^{3}$ Department of Nutritional Science, National Institute of Health and Nutrition, Tokyo, Japan \\ ${ }^{4}$ Faculty of Engineering, Chiba Institute of Technology, Narashino, Japan \\ ${ }^{5}$ Faculty of Informatics and Engineering, The University of Electro-Communications, Tokyo, Japan \\ ${ }^{6}$ National Institute of Health and Nutrition, Tokyo, Japan \\ ${ }^{7}$ Department of Health Promotion and Exercise, National Institute of Health and Nutrition, Tokyo, Japan \\ ${ }^{8}$ Department of Nutrition, Koshien University, Hyogo, Japan \\ ${ }^{9}$ Department of Nutritional Education, Kanagawa Institute of Technology, Kanagawa, Japan \\ ${ }^{10}$ Faculty of Sport and Health Science, Ritsumeikan University, Shiga, Japan \\ (Submitted 14 November 2012 - Final revision received 15 May 2013 - Accepted 16 May 2013 - First published online 10 July 2013)
}

\begin{abstract}
The objective of the present study was to investigate whether a previously reported apparent negative relationship between fat mass and daily physical activity in Japanese adult women would also be observed in Japanese adult men. The subjects were grouped into quartiles of BMI and body fat percentage (\%BF). The number of steps walked each day and the duration of light- to vigorous-intensity physical activity were assessed by an accelerometer over the same period of time as for the doubly labelled water experiment. The results showed that BMI negatively correlated with the number of steps and time spent in moderate-intensity physical activity, whereas \%BF showed a negative relationship with physical activity-related energy expenditure (PAEE)/body weight (BW) and physical activity level. The analysis of data using \%BF quartiles revealed that PAEE/BW decreased from the second quartile in which the BMI was $<25 \mathrm{~kg} / \mathrm{m}^{2}$. These observations are similar to those reported in our previous study in Japanese adult women. These cross-sectional studies cannot prove causality, and that obesity causes physical inactivity may be the case. However, the results of the present study provide information regarding which physical activity variables should be used in longitudinal studies.
\end{abstract}

Key words: Body composition: Physical activity: Doubly labelled water: Accelerometry: Japanese adult men

The prevalence of obesity is rising rapidly in most Asian countries, especially in Japan with an increase of $46 \%$ from $1976-80$ to $2000^{(1)}$. Specifically, the overall prevalence of overweight and obese adult men in Japan has increased gradually over the past 20 years, whereas the prevalence of overweight and obese adult women has not changed ${ }^{(2)}$. Obesity is commonly caused by an energy imbalance, with low total energy expenditure (TEE) possibly being a contributing factor in this process. While a negative relationship between obesity and daily physical activity has been reported ${ }^{(3,4)}$, the majority of studies have not observed this relationship ${ }^{(5,6)}$. Therefore, the relationship between BMI (body weight (BW, kg) divided by height squared $\left(\mathrm{m}^{2}\right)$ ) and daily physical activity still remains unclear.

In studies using the doubly labelled water (DLW) method, the most accurate method for measuring TEE in free-living conditions $^{(7,8)}$, the majority of Western adult populations showed no relationship between BMI and physical activity level (PAL, the ratio of TEE:RMR) ${ }^{(9-11)}$.

On the other hand, we have recently reported that Japanese adult women with higher fat deposition had higher body fat percentage $(\% \mathrm{BF})$ with an apparent lower PAL compared with those with lower fat deposition ${ }^{(12)}$. However, this association was not observed when women were categorised using BMI. In addition,

Abbreviations: \%BF, body fat percentage; BW, body weight; DLW, doubly labelled water; FM, fat mass; FFM, fat-free mass; PAEE, physical activity-related energy expenditure; PAL, physical activity level; TEE, total energy expenditure.

*Corresponding author: K. Ishikawa-Takata, fax +8133203 1731, email kazu@nih.go.jp 
step counts or time spent in moderate- or vigorous-intensity physical activity assessed by an accelerometer (i.e. the part composed of PAL) was also found to be lower in women with higher fat deposition $^{(12)}$.

However, Westerterp \& Goran ${ }^{(13)}$ using a meta-analysis of data of 290 adults from twenty-two DLW studies reported that a higher PAL was related to a lower fat mass (FM) only in men but not in women. This indicates that the relationship between physical activity and fat deposition may be different between the sexes. However, this relationship was not observed if data were stratified according to BMI.

The main aim of the present study was to examine whether the negative relationship that we observed between $\% \mathrm{BF}$ and daily physical activity in our previous cross-sectional study in Japanese adult women ${ }^{(12)}$ was also apparent in Japanese adult men. Information from the present study will help to understand the role of daily physical activity in the increased prevalence of obesity in adult males.

\section{Methods}

\section{Subjects}

Participants were recruited through health-care centres or at workplaces in three urban districts in central and western Japan, as described previously ${ }^{(12)}$. In each location, subjects were included for the study according to the following criteria: (1) in good health; (2) not involved in hard physical labour such as farming or athletics; (3) BMI $>18.5 \mathrm{~kg} / \mathrm{m}^{2}$; (4) living in their home prefecture 2 weeks before and during the study; (5) not on a weight-loss or treatment diet; (6) alcohol consumption $<40 \mathrm{~g} / \mathrm{d}$. The occupations of the participants were mainly salesmen, teachers, clerks and desk jobs, and some were unemployed. Overall, eighty-five male subjects aged 30-69 years were selected for the study. Over the entire assessment period, subjects were instructed to carefully maintain their normal daily activities and eating patterns and to make no conscious effort to lose or gain weight. The present study was conducted according to the guidelines laid down in the Declaration of Helsinki, and all procedures involving human subjects were approved by the Ethical Committee of the National Institute of Health and Nutrition in Japan. Written informed consent was obtained from all subjects.

\section{Experimental procedures}

The experimental design has been described in detail in our previous study ${ }^{(12)}$. Briefly, urine samples were collected and BW and height measured on the day before the assessment of physical activity (day 0). RMR was measured in the supine position using a Douglas bag in the early morning $12 \mathrm{~h}$ or longer after the last meal. A single dose of DLW was then given orally to each subject. After administration of this dose, the participants were instructed to collect urine samples on the following day and at eight additional times at the same time of the day during the 2-week study period. On day 15 , BW and height were measured again to examine changes in BW during the study period. The subjects were then provided with an accelerometer and a self-administered diet history questionnaire. Daily physical activity was estimated over the same 2-week study period under free-living conditions using the DLW method and accelerometer.

\section{Measurement of energy expenditure and body composition}

We measured TEE using the DLW method, as described previously ${ }^{(12)}$. Briefly, the single dose of DLW consisted of approximately $0.06 \mathrm{~g} / \mathrm{kg}$ BW of ${ }^{2} \mathrm{H}_{2} \mathrm{O}$ (99.8 atom\%; Cambridge Isotope Laboratories) and $1.4 \mathrm{~g} / \mathrm{kg} \mathrm{BW}$ of $\mathrm{H}_{2}{ }^{18} \mathrm{O}(10 \cdot 0$ atom\%; Taiyo Nippon Sanso). Isotopic enrichment of urine samples was measured using an isotope ratio mass spectrometer (model DELTA Plus; Thermo Electron Corporation). The ${ }^{2} \mathrm{H}$ and ${ }^{18} \mathrm{O}$ zero-time intercepts and elimination rates $\left(k_{\mathrm{H}}\right.$ and $\left.k_{\mathrm{O}}\right)$ were calculated using least-squares linear regression of the natural logarithm of the isotope concentration as a function of the elapsed time from dose administration. The zero-time intercepts were used to determine isotope pool sizes. Total body water was calculated as the mean value of the isotope pool size of ${ }^{2} \mathrm{H}$ divided by 1.041 and that of ${ }^{18} \mathrm{O}$ divided by 1.007 . Fat-free mass (FFM) was calculated by assuming a hydration of $0.732^{(14)}$. FM was calculated as BW minus FFM, while \%BF was computed from BW and FFM. Calculation of TEE $(\mathrm{kJ} / \mathrm{d})$ was performed using the modified Weir formula ${ }^{(15)}$ based on the $\mathrm{CO}_{2}$ production rate and respiratory quotient. Food quotient calculated from the diet history questionnaire was used instead of the respiratory quotient. This assumes that under conditions of perfect nutrient balance, the food quotient must equal the respiratory quotient ${ }^{(16,17)}$. PAL was estimated by dividing TEE by RMR. Physical activity-related energy expenditure (PAEE) was calculated as $0.9 \times$ TEE - RMR, assuming that the thermic effect of food was $10 \%$ of $\mathrm{TEE}^{(18)}$.

\section{Other measurements}

Measurements of anthropometry, accelerometry and the diet history questionnaire have been described in detail in our previous study $^{(12)}$. Briefly, a uniaxial accelerometer (Lifecorder EX; Suzuken Company Limited) was used for measuring the intensity of physical activity and step counts. The intensity of physical activity every 2 min was classified as either light ( $<3$ metabolic equivalents), moderate ( 3 to $<6$ metabolic equivalents) or vigorous ( $\geq 6$ metabolic equivalents) ${ }^{(16)}$. The validation and methodology of the diet history questionnaire have been described in detail elsewhere ${ }^{(17)}$.

\section{Statistical analysis}

Data are presented as means and standard deviations. BMI was calculated as BW (measured before the DLW dose) divided by height squared $\left(\mathrm{kg} / \mathrm{m}^{2}\right)$. The associations between physical activity and $\mathrm{BMI}$ or $\% \mathrm{BF}$, expressed as continuous variables, were examined by the linear regression analysis with or without adjustment for covariates. Subjects were then grouped according to quartiles of BMI and \%BF. A oneway ANOVA or ANCOVA was used to compare the variables in the quartiles, with Fisher's least-square difference used as

\footnotetext{
with an accelerometer and a self-administered diet history
} 
Table 1. Participants' characteristics, energy expenditure components and physical activity variables, grouped according to BMI\|

(Mean values and standard deviations)

\begin{tabular}{|c|c|c|c|c|c|c|c|c|c|c|}
\hline \multirow[t]{2}{*}{ BMI quartiles... } & \multicolumn{2}{|c|}{ First $\left(18 \cdot 7-22.5 \mathrm{~kg} / \mathrm{m}^{2}\right)$} & \multicolumn{2}{|c|}{$\begin{array}{c}\text { Second } \\
\left(22.6-24.5 \mathrm{~kg} / \mathrm{m}^{2}\right)\end{array}$} & \multicolumn{2}{|c|}{$\begin{array}{c}\text { Third } \\
\left(24.6-27.5 \mathrm{~kg} / \mathrm{m}^{2}\right)\end{array}$} & \multicolumn{2}{|c|}{$\begin{array}{c}\text { Fourth } \\
\left(27 \cdot 6-39 \cdot 1 \mathrm{~kg} / \mathrm{m}^{2}\right)\end{array}$} & \multirow[b]{2}{*}{$P$ (ANOVA) } & \multirow[b]{2}{*}{ П } \\
\hline & Mean & SD & Mean & SD & Mean & SD & Mean & SD & & \\
\hline \multicolumn{11}{|l|}{ Energy expenditure } \\
\hline TEE $(k J / d)\|\|$ & 11070 & 1290 & 11663 & 2055 & 12188 & 1949 & $13396+† \neq \ddagger \S$ & 2172 & 0.009 & $0.458^{\star \star}$ \\
\hline RMR (kJ/d) & 5629 & 769 & 5792 & 774 & 6039 & 809 & $7165 \dagger † \ddagger \S \S$ & 861 & $<0.001$ & $0.658^{\star *}$ \\
\hline PAL & 1.99 & 0.23 & $2 \cdot 02$ & 0.29 & 2.03 & 0.29 & 1.87 & 0.19 & 0.227 & -0.168 \\
\hline PAEE $(k J / d)$ & 4335 & 957 & 4705 & 1521 & 4930 & 1454 & 4891 & 1390 & 0.830 & 0.169 \\
\hline PAEE/BW (kJ/d per kg) ๆ ๆ & $71 \cdot 7$ & $16 \cdot 9$ & $69 \cdot 0$ & $21 \cdot 8$ & 67.4 & $18 \cdot 9$ & $52 \cdot 9 \dagger † £ \S$ & $12 \cdot 2$ & 0.008 & $-0.343^{\star \star}||||||$ \\
\hline \multicolumn{11}{|l|}{ Accelerometer } \\
\hline Step counts (per d) & 10296 & 2699 & 10085 & 2891 & 9848 & 3001 & $7992 \dagger \ddagger \S$ & 3222 & 0.027 & $-0.259^{\star}$ \\
\hline Light, <3 MET (min/d) & $56 \cdot 6$ & $12 \cdot 5$ & $55 \cdot 1$ & $16 \cdot 2$ & $57 \cdot 6$ & $20 \cdot 2$ & 54.9 & $21 \cdot 4$ & 0.695 & -0.017 \\
\hline Moderate, 3 to < $6 \mathrm{MET}(\mathrm{min} / \mathrm{d})$ & 39.8 & $17 \cdot 9$ & 37.5 & $12 \cdot 9$ & $35 \cdot 8$ & $19 \cdot 6$ & $24 \cdot 2 \dagger \dagger \neq \ddagger \S$ & $14 \cdot 3$ & 0.009 & $-0.298^{\star \star}$ \\
\hline Vigorous, $6 \mathrm{MET}(\mathrm{min} / \mathrm{d})$ & 3.6 & $3 \cdot 0$ & 4.7 & $6 \cdot 6$ & $3 \cdot 2$ & $4 \cdot 0$ & $2 \cdot 2$ & 4.6 & 0.397 & -0.154 \\
\hline
\end{tabular}

TEE, total energy expenditure; PAL, physical activity level (TEE/RMR); PAEE, physical activity-related energy expenditure $(0.9 \times$ TEE - RMR); BW, body weight; MET, metabolic equivalents.

There was a significant correlation with $\mathrm{BMI}:{ }^{*} P<0.05,{ }^{* *} P<0.01$.

Mean values were significantly different from those of the first quartile: $\dagger P<0.05$, t† $P<0.01$

Mean values were significantly different from those of the second quartile: $\ddagger P<0.05$.

Mean values were significantly different from those of the third quartile: $\S P<0.05$

\| The subjects were categorised by quartile. The number of subjects in each quartile was twenty-five. In the nine intensity levels (1-9) of physical activity assessed by using an accelerometer, $1-3$ indicates light intensity,

I The associations between BMI and physical activity variables and energy expenditure components, expressed as continuous variables, were examined by the linear regression analysis.

IIIIA statistical significance in TEE among the quartiles was still found even after adjustment for BW using ANCOVA $(P=0.026)$

19 There was no significant difference in PAEE among the quartiles after adjustment for BW using ANCOVA $(P=0.155)$.

\|\|\|\| There was no significant difference between BMI and PAEE after adjustment for BW (partial $r-0.046, P=0.677$ ). 
a post hoc test for multiple comparisons. Differences were considered statistically significant when the $P$ value was $<0 \cdot 05$. All statistical analyses were carried out using SPSS for Windows (version $16.0 \mathrm{~J}$; SPSS, Inc.).

\section{Results}

Of the total eighty-five men studied, the proportion of normalweight (BMI 18.5 to $<25 \mathrm{~kg} / \mathrm{m}^{2}$ ), overweight (BMI $\geq 25$ to $<30 \mathrm{~kg} / \mathrm{m}^{2}$ ) and obese participants $\left(\mathrm{BMI} \geq 30 \mathrm{~kg} / \mathrm{m}^{2}\right.$ ) was $54 \cdot 1,35 \cdot 3$ and $10.6 \%$, respectively. The mean age of the subjects was 47.5 (SD 11.0, range 30-69) years. The mean BW, height and BMI were 73.5 (SD $14 \cdot 0$, range $51 \cdot 1-116 \cdot 3) \mathrm{kg}$, 1.70 (SD 0.07, range, $1.51-1.88$ ) $\mathrm{m}$ and 25.3 (SD 3.9), range $18 \cdot 7-39 \cdot 1) \mathrm{kg} / \mathrm{m}^{2}$, respectively. BW did not change during the study (change in BW: -0.13 (sD 0.04$) \mathrm{kg}, P=0.987$ ). The range of PAL was $1.46-2.51$ with a mean value of 1.98 .

The relationships between BMI and energy expenditure or physical activity variables are shown in Table 1 and Fig. 1. Both FM ( $r$ 0.824) and FFM ( $r$ 0.714) showed a linear correlation with BMI. TEE increased linearly with BMI, whereas PAEE/BW, step counts and moderate-intensity physical activity showed a negative correlation. PAL and PAEE adjusted for BW did not correlate with BMI (Fig. 1).

Energy expenditure and physical activity variables were also compared between the BMI quartiles. Age and height were not significantly different between the quartiles. TEE, step counts and moderate-intensity physical activity were significantly different between the quartiles. The fourth quartile had significantly smaller PAEE/BW, step counts and moderate-intensity PAL compared with the other three quartiles. A statistically significant decrease in TEE between the BMI quartiles was still observed after adjustment for BW using ANCOVA $(P=0.026)$. However, PAEE did not show any significant difference between the BMI quartiles after adjustment for BW using ANCOVA $(P=0 \cdot 155)$.

$\mathrm{PAL}, \mathrm{PAEE} / \mathrm{BW}$ and moderate-intensity physical activity negatively correlated with \%BF (Table 2; Fig. 1). FM increased with \%BF ( $r$ 0.876), whereas FFM did not ( $r$ 0.008). As shown in Fig. 1, after PAEE was adjusted for BW, a negative correlation was still observed between PAEE and \%BF. A statistically significant difference was found between the $\% \mathrm{BF}$ quartiles for PAL, PAEE/BW, step counts and moderate- and vigorousintensity physical activities. A significant difference in TEE and PAEE was still found between the \%BF quartiles after adjustment for BW using ANCOVA $(P=0.001$ and 0.002). PAEE was lower in the second and fourth quartiles than in the first quartile when it was adjusted for BW using ANCOVA. PAL was lower in the second and fourth quartiles than in the first quartile, whereas there was no significant difference between the second and fourth quartiles.

\section{Discussion}

The present study aimed at comparing the relationship of BMI and $\% \mathrm{BF}$ with various aspects of daily physical activity between Japanese adult men investigated in the present study and Japanese adult women that we have reported
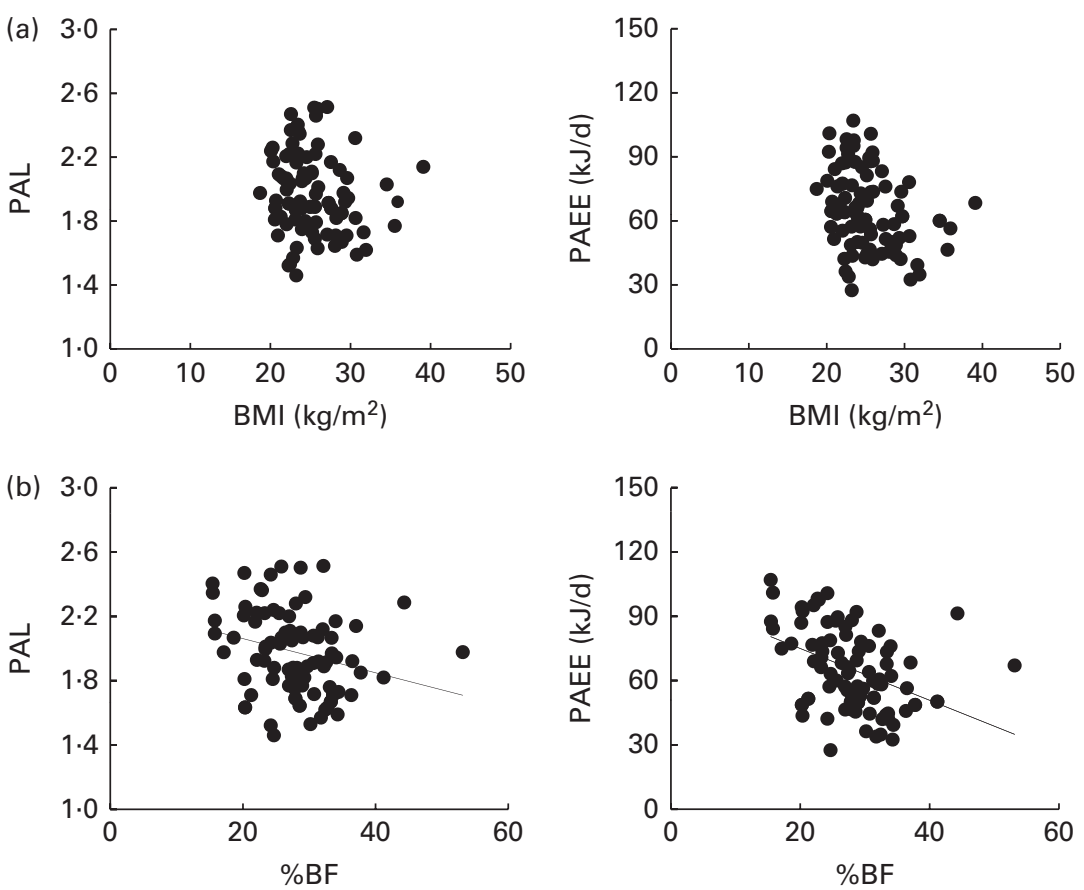

Fig. 1. Relationships between (a) BMI and physical activity level (PAL) or physical activity-related energy expenditure (PAEE) and (b) between body fat percentage (\%BF) and PAL or PAEE. PAL = TEE/RMR, where TEE is the total energy expenditure; PAEE $=0.9 \times \mathrm{TEE}-\mathrm{RMR}$. \%BF was negatively associated with PAL or PAEE, after adjustment for body weight, although this relationship was not observed between BMI and PAL or PAEE even after adjustment for body weight. (a) PAL: $r-0.17$; PAEE: partial $r-0.05$ (adjusted for body weight). (b) PAL: $r-0.26, P<0.05$; PAEE: partial $r-0.27$ (adjusted for body weight), $P<0.05$. 
previously ${ }^{(12)}$. The common finding in both studies was that BMI was negatively related to the number of steps and time spent in moderate-intensity physical activity, whereas \%BF was negatively related to PAEE/BW and PAL.

No significant correlation was observed between BMI and PAL in Japanese adult men, a finding consistent with previous studies in Japanese women ${ }^{(12)}$ and Western populations ${ }^{(9-11)}$. On the other hand, Tooze et al. ${ }^{(19)}$ reported that normal (BMI $<25 \mathrm{~kg} / \mathrm{m}^{2}$ ) and overweight (BMI 25 to $<30 \mathrm{~kg} / \mathrm{m}^{2}$ ) women had a higher PAL compared with obese women (BMI $\geq 30 \mathrm{~kg} / \mathrm{m}^{2}$ ). This relationship was not observed in adult men in their study.

However, grossly obese subjects often showed a lower PAL. Prentice et $a l .{ }^{(9)}$ reported that grossly obese men (BMI $\geq 35 \mathrm{~kg} / \mathrm{m}^{2}$ ) tended to have a lower PAL (mean 1.52 ) compared with normal-weight and obese men (BMI 18.5 to $<35 \mathrm{~kg} / \mathrm{m}^{2}$ ) whose mean PAL values ranged between 1.80 and 1.86 . In the present study, the number of grossly obese men was three and their PAL values were 1.77, 1.94 and $2 \cdot 14$, respectively. Therefore, the lower mean PAL value of 1.52 reported by Prentice et $a l .{ }^{(9)}$ was not observed in grossly obese men in the present study. Similarly, Das et al. ${ }^{(11)}$ reported that PAL did not differ in grossly obese women within the BMI range of $37.5-77 \mathrm{~kg} / \mathrm{m}^{2}$.

In contrast to the lack of the relationship between BMI and PAL, we showed that PAEE/BW was significantly lower in the highest quartile of BMI in Japanese adult men, a result similar to that observed in Japanese women ${ }^{(12)}$. This result is also in agreement with the study by Schoeller ${ }^{(20)}$, which reported that obese Western populations had a lower PAEE/BW compared with non-obese populations. However, the present study revealed that there was no significant relationship between BMI and PAEE, following adjustment for BW as a covariate. Despite the convenience of correcting for body size, dividing PAEE by BW should be interpreted with caution, as this may be subject to artifacts due to problems with a zero intercept or the requirement of proportionality.

Although no significant relationship was observed between BMI and PAEE after correction for body size, \%BF negatively correlated with both PAL and PAEE/BW. In particular, a significant relationship was still found between \%BF and PAEE even after adjustment for BW as a covariate. The present study also showed a similar relationship between PAEE/BW and \%BF to that observed in women ${ }^{(12)}$, Chinese adults ${ }^{(21)}$ and Western men $^{(22)}$. Therefore, it is not necessary to consider ethnic differences in this relationship. Moreover, when the data were analysed using the \%BF quartiles (Table 2), PAEE/BW decreased from the second quartile in which BMI was $<25 \mathrm{~kg} / \mathrm{m}^{2}$. These results are similar to those observed in Japanese women $^{(12)}$, and suggested that daily physical activity differed according to the level of fat deposition, even in the normal-weight adult population. However, it is also important to note that PAEE corrected for BW has the limitation of assuming that all physical activities are weight-dependent.

The present study showed the negative relationship between BMI and step counts or moderate-intensity physical activity, which is likely to that observed in Japanese women. However, Mitsui et al. ${ }^{(23)}$ reported that there was a significant 
relationship between step counts and BMI in Japanese adult women, but not in men. This discrepancy in the relationship between BMI and step counts in Japanese subjects may be attributable to lower BW, BMI and step counts in the study by Mitsui et al. ${ }^{(23)}$. Thus, physical activity variables assessed by an accelerometer may be useful for understanding daily physical activity in obese adult populations in large epidemiological studies.

On the other hand, time spent in moderate-intensity physical activity was significantly related to $\% \mathrm{BF}$ in men, although this relationship was not observed in women ${ }^{(12)}$. Conversely, the number of steps was related to \% $\mathrm{BF}$ not in men but in women. The reason for this discrepancy between the sexes was not clear, although it is possible that $\% \mathrm{BF}$ may be related to the total energy expended in daily physical activity rather than to the part composed of PAEE or PAL that included the number of steps and exercise intensity.

The present study is limited by the cross-sectional study design, which makes it difficult to clarify the causality between obesity and physical inactivity. Therefore, we cannot exclude the possibility of reverse causality that obesity may cause physical inactivity. For example, obese subjects fatigue after taking a few steps because they have to propel a larger mass; as a result, the lowered physical activity may cause obesity $^{(24)}$. In line with this, men in the fourth quartile, almost obese, may have had less volitional movement during physical activity due to their excess weight. The possibility of reversal causality is in agreement with the longitudinal study by Luke et $a l{ }^{(25)}$ demonstrating that physical inactivity on the basis of energy expenditure does not predict weight change.

The present study also has the following limitations. First, FFM hydration was assumed to be equal in all participants at a value of $0.732^{(14)}$; therefore, some errors in estimating FM obtained from FFM may have resulted from differences in the levels of obesity and sex. Second, PAL in the study was higher than $1 \cdot 75$, the value reported in the general population of Eastern and Western countries ${ }^{(21,26-28)}$. The average daily number of steps of about 9564 observed in the participants of the present study was also higher than that reported for Japanese adult men in another study, who generally walk an average of 7893 steps/ $\mathrm{d}^{(29)}$. This indicates that the individuals included in the present study may have been more physically active than adult men in the general Japanese population. However, the ranges of PAL were similar between men and women (1.46-2.51 and 1.36$2 \cdot 52$, respectively $)^{(12)}$, which were within the PAL of the general population $^{(30)}$

In conclusion, the present cross-sectional study in Japanese adult men showed a negative relationship between BMI and the number of steps and time spent in moderate-intensity physical activity. It also showed that men with higher fat deposition were less active on the basis of PAEE adjusted for BW and PAL. These observations are very similar to those reported in our previous study in adult Japanese women ${ }^{(12)}$. These cross-sectional studies cannot prove causality, and that obesity causes physical inactivity may be the case. Despite these limitations, the present study did provide information regarding which physical activity variables are appropriate for use in a longitudinal study. Additional research using a longitudinal study design is required to examine the cause-effect relationships between obesity and physical inactivity including factors of dietary intake.

\section{Acknowledgements}

The present study was performed as part of Health and Labor Sciences Research Grants (Comprehensive Research on Cardiovascular and Life-Style Related Diseases) from the Ministry of Health, Labor and Welfare of Japan. The study was supported by the SMART Research Professor Program of Konkuk University. We thank the staff of the National Institute of Health and Nutrition for their kind cooperation. J. P. analysed and interpreted the data and wrote the manuscript. K. I.-T. interpreted the data, wrote the manuscript and had primary responsibility for the final content. S. T., Y. H. and K. O. interpreted the data. S. W., M. M., A. M. and N. A. managed the field study. I. T. managed the study. All authors approved the final version of the manuscript. The authors declare that they have no conflicts of interest. The authors have no relevant financial interest in this article.

\section{References}

1. Asia Pacific Cohort Studies Consortium (2006) The burden of overweight and obesity in the Asia-Pacific region. Obes Rev 8, 191-196.

2. Yoshiike N, Seino F, Tajima S, et al. (2002) Twenty-year changes in the prevalence of overweight in Japanese adults: the National Nutrition Survey 1976-95. Obes Rev 3, 183-190.

3. Schoeller DA (1998) Balancing energy expenditure and body weight. Am J Clin Nutr 68, 956S-961S.

4. Yoshioka M, Doucet E, St-Pierre S, et al. (2001) Impact of high-intensity exercise on energy expenditure, lipid oxidation and body fatness. Int J Obes Relat Matab Disord 25 , 332-339.

5. Goran MI (2000) Energy metabolism and obesity. Med Clin North Am 84, 347-362.

6. Luke A, Dugas LR, Ebersole K, et al. (2009) Energy expenditure does not predict weight change in either Nigerian or African American women. Am J Clin Nutr 89, 169-176.

7. Schoeller DA (1999) Recent advances from application of doubly labeled water to measurement of human energy expenditure. J Nutr 129, 1765-1768.

8. Schoeller DA (2008) Insights into energy balance from doubly labeled water. Int J Obes 32, S72-S75.

9. Prentice AM, Black AE, Coward WA, et al. (1996) Energy expenditure in overweight and obese adults in affluent societies: an analysis of 319 doubly-labeled water measurements. Eur J Clin Nutr 50, 93-97.

10. Butte NF, Treuth MS, Mehta NR, et al. (2003) Energy requirements of women of reproductive age. Am J Clin Nutr 77, 630-638.

11. Das SK, Saltzman E, McCrory MA, et al. (2004) Energy expenditure is very high in extremely obese women. $J$ Nutr 134, 1412-1416.

12. Park J, Ishikawa-Takata K, Tanaka S, et al. (2011) Relation of body composition to daily physical activity in free-living Japanese adult women. Br J Nutr 106, 1117-1127.

13. Westerterp KR \& Goran MI (1997) Relationship between physical activity related energy expenditure and body 
composition: a gender difference. Int J Obes Relat Metab Disord 21, 184-188.

14. Pace N \& Rathbun EN (1945) Studies on body composition III. The body water and chemically combined nitrogen content in relation to fat content. J Biol Chem 158, 685-691.

15. Weir JBdV (1949) New methods for calculating metabolic rate with special reference to protein metabolism. J Physiol 109, 1-9.

16. Kumahara H, Schutz Y, Ayabe M, et al. (2004) The use of uniaxial accelerometry for the assessment of physicalactivity-related energy expenditure: a validation study against whole-body indirect calorimetry. Br J Nutr 91, 235-243.

17. Sasaki S, Yanagibori R \& Amano K (1998) Self-administered diet history questionnaire developed for health education: a relative validation of the test-version by comparison with 3-day diet record in women. $J$ Epidemiol 8, 203-215.

18. Reed GW \& Hill JO (1996) Measuring the thermic effect of food. Am J Clin Nutr 63, 164-169.

19. Tooze JA, Schoeller DA, Subar AF, et al. (2007) Total daily energy expenditure among middle-aged men and women: the OPEN study. Am J Clin Nutr 86, 382-387.

20. Schoeller DA (1998) Balancing energy expenditure and body weight. Am J Clin Nutr 68, 956S-961S.

21. Schulz LO \& Schoeller DA (1994) A compilation of total daily energy expenditures and body weights in healthy adults. $\mathrm{Am}$ J Clin Nutr 60, 676-681.
22. Yao M, McCrory MA, Ma G, et al. (2003) Relative influence of diet and physical activity on body composition in urban Chinese adults. Am J Clin Nutr 77, 1409-1416.

23. Mitsui T, Shimaoka K, Tsuzuku S, et al. (2008) Pedometerdetermined physical activity and indicators of health in Japanese adults. J Physiol Anthropol 27, 179-184.

24. Levine JA, Lanningham-Foster LM, McCrady SK, et al. (2005) Interindividual variation in posture allocation: possible role in human obesity. Science 307, 584-586.

25. Luke A, Dugas LR, Ebersole K, et al. (2009) Energy expenditure does not predict weight change in either Nigerian or African American women. Am J Clin Nutr 89, 169-176.

26. Black AE, Prentice AM \& Coward WA (1986) Use of food quotients to predict respiratory quotients for the doubly labeled water method of measuring energy expenditure. Hum Nutr Clin Nutr 40C, 381-391.

27. Westerterp KR (1999) Obesity and physical activity. Int J Obes 23, 59-64.

28. Ishikawa-Takata K, Tabata I, Sasaki S, et al. (2008) Physical activity level in healthy free-living Japanese estimated by doubly-labeled water method and International Physical Activity Questionnaire. Eur J Clin Nutr 62, 885-891.

29. Ministry of Health, Labour and Welfare, Japan (2007) The National Health and Nutrition Survey in Japan. Tokyo: Daiichi Shuppan (in Japanese).

30. Westerterp KR (1998) Alterations in energy balance with exercise. Am J Clin Nutr 68, 970S-974S. 